\title{
Penetration of Topically Applied Prednisolone Sodium Phosphate into Human Aqueous Humour
}

\author{
C. N. J. McGHEE,,$^{1.3}$ M. J. NOBLE, ${ }^{2}$ D. G. WATSON,${ }^{3}$ G. N. DUTTON, ${ }^{3}$ A. I. FERN, ${ }^{1}$ \\ T. M. HEALEY ${ }^{3}$ and J. M. MIDGLEY ${ }^{3}$ \\ Glasgow
}

\begin{abstract}
Summary
Gas Chromatography with Mass Spectrometry (GCMS) was utilised to determine the penetration of prednisolone sodium phosphate into the aqueous humour of human volunteers undergoing routine cataract extraction. Detectable levels of prednisolone were measured in the aqueous humour within 15 minutes. Peak concentrations occurred between 90 and 240 minutes, and the steroid could not be detected in samples taken 10 hours or more after topical administration.
\end{abstract}

Topical corticosteroids have long been used for a variety of ophthalmic conditions. Despite their widespread use, treatment regimens depend largely on clinical experience and the conflicting results obtained by animal experimentation (1-15). Indeed, until recently little information has been available on the absorption of topically applied corticosteroids in human aqueous humour (16). The authors have established the sensitivity and specificity of Gas Chromatography with Mass Spectrometry (GCMS) in the measurement of dexamethasone alcohol in human aqueous humour and have utilised this technique to measure accurately the penetration of topically applied prednisolone sodium phosphate. A commercially available preparation of prednisolone sodium phosphate 0.5 per cent (Predsol: Glaxo Laboratories, UK) was used and this report forms part of a larger study, investigating the ocular pharmacokinetics in humans, of the commercially prepared ophthalmic corticosteroids which are com- monly used by ophthalmologists. It is hoped that the final results of this series might provide a more rational basis for the treatment regimens of topical ophthalmic steroids.

\section{Subjects and Methods}

Volunteers were obtained from patients undergoing routine cataract surgery and informed consent was obtained in all cases. Patients who had corneal disease, inflammatory eye conditions, previous ocular surgery or who had been on oral or topical corticosteroids during the month prior to surgery were excluded. Samples of aqueous humour obtained from 93 eyes of 93 patients were included in the study.

Fifty microlitres of prednisolone sodium phosphate 0.5 per cent (Predsol, Glaxo) were introduced by micropipette into the lower conjunctival fornix of the eyes being prepared for surgery at predetermined times pre-operatively. These eyes were also treated with topically applied phenylephrine (10 per cent),

\section{From:}

'Ophthalmology Department, Southern General Hospital, Govan Road, Glasgow G51 4TF.

${ }^{2}$ Tennent Institute of Ophthalmology, University of Glasgow, Western Infirmary, Glasgow G11.

${ }^{3}$ Department of Pharmacy, University of Strathclyde, Glasgow G1 1XW.

Correspondence to: Dr. McGhee, Southern General Hospital, Govan Road, Glasgow G51 4TF. 
and cyclopentolate ( 1 per cent) one hour preoperatively to produce mydriasis.

Fifty-nine of the operations were performed under general anaesthesia and 34 under retrobulbar anaesthesia. The patient age range was 57-91 years ( mean $=73$ yrs.) During cataract surgery, after fashioning a partial thickness corneo-scleral incision, the anterior chamber was entered with a 25 gauge needle on a tuberculin syringe and 100-150 microlitres of aqueous humour were aspirated. The precise time interval between instillation of the topical prednisolone sodium phosphate and the aqueous sampling was noted (range 6-1115 mins.) The aqueous samples were stored at -20 degrees $C$ and subsequently analysed by GCMS.

Tetradeuterated prednisolone (1 ng) (17) was added to the sample of aqueous humour (which had previously been diluted to one millilitre with high purity water) as an internal standard. The aqueous layer was extracted with ethyl acetate $(2 \times 1 \mathrm{ml})$ and the extract chemically treated to provide the dimethoxime trimethylsilylether (MOTMS) derivative (17). The derivatised extract was then subjected to GCMS in the negative ion chemical ionisation mode. The undeuterated corticosteroid present in the aqueous humour was identified unequivocally and quantified with reference to the known amount of tetradeuterated prednisolone added before the extraction procedure. The MOTMS derivative of the latter co-chromatographed with that of the corresponding undeuterated sub-

Table I. Mean concentration of prednisolone $(\mathrm{ng} / \mathrm{ml}$ $+/-$ standard error) in human aqueous humour at various times ( $\mathrm{min}$ ) following topical administration of prednisolone sodium phosphate $(0.5$ per cent $)$.

\begin{tabular}{ccccc}
\hline & & \multicolumn{3}{c}{$\begin{array}{c}\text { Mean Concentration in } \\
\text { Aqueous Humour }\end{array}$} \\
\cline { 3 - 5 } $\begin{array}{c}\text { Time Interval } \\
\text { (mins) }\end{array}$ & $\begin{array}{c}\text { No. of Eyes } \\
(n=93)\end{array}$ & $\begin{array}{c}\text { (ng/ml) } \\
\text { (n) }\end{array}$ & $+/-$ & $($ SEM) \\
\hline $0-30$ & 12 & 4.3 & $+/-$ & 0.92 \\
$31-60$ & 18 & 7.3 & $+/-$ & 0.97 \\
$61-90$ & 8 & 13.7 & $+/-$ & 2.4 \\
$91-120$ & 10 & 25.6 & $+/-$ & 4.0 \\
$121-180$ & 16 & 24.9 & $+/-$ & 2.1 \\
$181-240$ & 11 & 22.9 & $+/-$ & 5.6 \\
$241-480$ & 9 & 8.7 & $+/-$ & 2.1 \\
$650-850$ & 9 & 0.0 & $+/-$ & 0.0 \\
\hline
\end{tabular}

stance and yielded ions in its mass spectrum equivalent to those obtained from the undeuterated compound but with a mass increment of $4 \mathrm{amu}$. This method is suitable for the accurate quantification of prednisolone in the $0.1-10$ nanogram range.

To determine whether or not any prednisolone sodium phosphate remained dissolved in the aqueous humour after extraction, the remaining sample was incubated in sodium acetate buffer containing acid phosphatase (ex wheatgerm: Sigma Chemical Ltd.) This produced no further detectable amounts of prednisolone when the sample was analysed in the manner described above.

\section{Results}

The concentrations of prednisolone in human aqueous humour during successive time intervals following the topical administration of $50 \mu \mathrm{l}$ of prednisolone sodium phosphate $(0.5$ per cent) are shown in Table I. The highest mean concentrations were found between 90 and 240 mins. Subsequently the intra-ocular levels of the steroid diminished and the steroid could not be detected in aqueous samples taken ten or more hours after topical application. Variations in the concentrations of prednisolone in human aqueous humour with time from application are shown in Figure 1.

\section{Discussion}

Previous studies of the penetration of topically applied corticosteroids into the aqueous humour have mainly used the rabbit model; in vivo $(1-7,10,12,13,14)$ and in vitro (8). More recently, isolated bovine eyes have been utilised in penetration studies (15). The initial studies employed relatively insensitive techniques (1-3), whereas the majority of later investigations have utilised radio-labelling of the steroid nucleus $(4-8,10,13-15)$. These animal studies have provided variable results for peak aqueous humour concentrations (140-9000 $\mathrm{ng} / \mathrm{ml})$ following topical administration of prednisolone sodium phosphate $(0.125$ per cent -1.0 per cent $)(6,10)$ and prednisolone acetate $(0.1$ per cent-1.0 per cent $)(4,12,13)$ to uninflamed eyes with intact corneal epithelium. Both derivatives of prednisolone afforded higher 


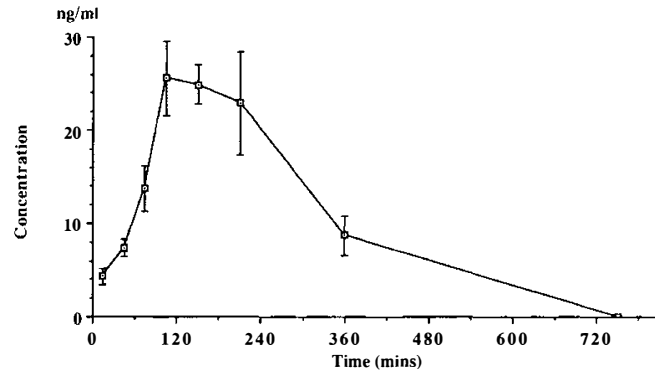

Fig. 1. Concentration of prednisolone ( $\mathrm{ng} / \mathrm{ml}+1-$ standard error) in human aqueous humour vs time (mins) following topical administration of prednisolone sodium phosphate (0.5 per cent).

concentrations of steroid in the aqueous humour when presented in a concentration of 1 per cent as compared to 0.1 per cent concentrations and it has been suggested by Leibowitz and Kupferman (1976) that the prednisolone acetate 1 per cent formulation provides the optimal dose response curve when compared to higher or lower concentrations of the same drug (14). Maximal aqueous humour levels of prednisolone have been recorded in rabbits within 90 mins of instillation of prednisolone sodium phosphate $(6,10)$. The very marked variation in peak concentrations of prednisolone which have been recorded in rabbit aqueous humour may reflect the limitations of radio-labelling techniques since only the radiolabelled fraction is measured. This technique does not provide information concerning the chemical nature of the label (i.e. whether this is attached to the intact steroid or a metabolite, unless additional chromatographic techniques are employed) and, most importantly, the sensitivity of the method depends upon the amount of radiolabel used (18). The ability of the cornea to metabolise steroids enzymically highlights the importance of recognising whether intact steroids or their metabolites are actually being measured (19).

The technique of GCMS has several advantages over previous techniques: it is extremely sensitive and allows the accurate and specific measurement of nanogram per millilitre levels of corticosteroids (16). The specificity of this technique allows the intact prednisolone molecule to be distinguished from its metabolites and, since labelling of the steroid is not required, commercial preparations of a drug can be studied unaltered.

Although the methodology per se does not permit a distinction to be made between prednisolone and prednisolone sodium phosphate, the latter is highly water soluble and therefore unlikely to be extracted from the aqueous humour into an organic solvent such as ethyl acetate. Subsequent treatment of the aqueous humour with phosphatase, followed by re-extraction and analysis, did not afford any additional prednisolone, which leads to the conclusion that the steroid is present in the aqueous humour as the free alcohol. However, it is not possible to ascertain whether or not it is absorbed in this form or as the phosphate ester, although absorption of the sodium salt of the latter appears unlikely on purely physicochemical grounds.

We found that prednisolone sodium phosphate or prednisolone itself penetrated the cornea rapidly and detectable levels of prednisolone were recorded in the aqueous humour at 13-15 minutes post topical application. Mean peak concentrations were noted in the 90-240 minute time interval and significant levels were recorded up to eight hours after application. However, the drug could not be detected 10 hours after topical application. The mean peak level $(25.6 \mathrm{ng} / \mathrm{ml})$ is considerably less than is reported in previous animal studies $(6,10)$, although the time taken to reach the initial peak concentration is not dissimilar. This difference may merely reflect differences between the techniques used for measurement but, more probably, it may be due to the known anatomical and physiological differences between the human cornea and the thinner rabbit corneoscleral envelope. It is notable that while earlier work has suggested that the lipophilic corneal epithelium presents a significant barrier to the absorption of steroid from preparations of prednisolone sodium phosphate, it has been shown to penetrate the intact epithelium in higher concentrations than would be theoretically suggested (10). Prednisolone sodium phosphate solution has also been shown to penetrate the cornea by a factor of up to a twofold when compared to prednisolone in $\mathrm{NaCl}$ buffer, thus emphasising the effect of the vehicle on absorption of the 
steroid $(6,10)$. In the present study the intact corneal epithelium did not appear to present a significant barrier to rapid absorption although it may have exerted an influence on the peak concentrations obtained. Certainly the available animal data suggests that the biphasic prednisolone acetate, which is more lipophilic, achieves higher concentrations in aqueous humour than prednisolone sodium phosphate in the presence of the intact corneal epithelium $(4,12,13)$. Furthermore prednisolone sodium phosphate (1 per cent) has markedly less anti-inflammatory action on the intact inflamed cornea when compared to prednisolone acetate ( 1 per cent) (9). However, in the absence of the epithelium there is no statistical difference between the antiinflammatory potencies of these two preparations (11). When the present results are compared with those from our previous study (16) on the penetration of dexamethasone alcohol ( 0.1 per cent $)$ it is notable that both prednisolone and dexamethasone are measurable by GCMS in the aqueous humour within 30 mins of application and that peak levels occur within the period 90-240 mins post instillation. However, dexamethasone attained a higher mean peak level $(31.0 \mathrm{ng} / \mathrm{ml})$ when compared to prednisolone $(25.6 \mathrm{ng} / \mathrm{ml})$ and measurable amounts were present at 12 hours while no predisolone could be detected after 10 hours.

There are shortcomings in our investigations: the use of patients with cataract (whose eyes may not necessarily behave like nonpathological eyes), the preoperative application of mydriatics (which may or may not influence steroid absorption) and the unknown effects of general or retrobulbar anaesthesia (although there was no statistical difference in the recorded steroid levels in those who received retrobulbar anaesthesia when compared to those who had general anaesthesia). Unfortunately these are unavoidable factors inherent in such human studies.

The authors believe that this is the first study in which the absorption curve for a commercially available preparation of prednisolone sodium phosphate ( 0.5 per cent) has been established in human subjects. Further clinical studies based on the results described here might enable more rational therapeutic regimens for this preparation to be established.

We thank Miss Catherine Bates for technical assistance, Glaxo Group Research Ltd. and Merck Sharp \& Dohme Ltd. for gifts of synthetic corticosteroids and the Scottish Home and Health Department for providing funds for the research.

\section{References}

'Weimar VL and Leopold IH: Intraocular Penetration of Local Hydrocortisone and Cortisone. Arch Ophthalmol 1955, 52: 769-73.

${ }^{2}$ Leopold IH and Kroman HS: Methyl- and FluroSubstituted Prednisolones in the Blood and Aqueous Humor of the Rabbit. Arch Ophthalmol 1960, 63: 943-7.

${ }^{3}$ Kroman HS and Leopold IH: Studies upon Methyland Fluoro-substituted Prednisolones in the Aqueous Humor of the Rabbit. II. Chromatography. Am J Ophthalmol 1961, 52: 77-81.

${ }^{4}$ Murdick PW, Keates RH, Donovan EF, Wyman M, Short C: Ocular Penetration Studies. II. The Topical Administration of Prednisolone. Arch Ophthalmol 1966, 76: 602-3.

${ }^{5}$ Cox WV, Kupferman A, Leibowitz HM: Topically Applied Steroids in Corneal Disease. 1. The Role of Inflammation in Stromal Absorption of Dexamethasone. Arch Ophthalmol 1972, 88: 308-13.

${ }^{6}$ Green K and Downs SJ: Prednisolone Phosphate Penetration into and Through the Cornea. Invest Ophthalmol 1974, 13: 316-18.

${ }^{7}$ Kupferman A and Leibowitz HM: Topically Applied Steroids in Corneal Disease. IV. The Role of Drug Concentration in Stromal Absorption of Prednisolone Acetate. Arch Ophthalmol 1974, 91: $377-80$.

${ }^{8}$ Hull DS, Hine JE, Edelhauser HF, Hyndiuk RA: Permeability of the Isolated Rabbit Cornea to Corticosteroids. Invest Ophthalmol 1974, 13: 457-9.

${ }^{9}$ Leibowitz HM and Kupferman A: Anti-Inflammatory Effectiveness in the Cornea of Topically Administered Prednisolone. Invest Ophthalmol 1974, 13: 757-62.

10Kupferman A and Leibowitz HM: Topically Applied Steroids in Corneal Disease, VI. Kinetics of Prednisolone Sodium Phosphate. Arch Ophthalmol 1974, 92: 331-4.

"Kupferman A and Leibowitz HM: Anti-Inflammatory Effectiveness of Topically Administered Corticosteroids in the Cornea Without Epithelium. Invest Ophthalmol 1975, 14: 252-5.

${ }^{12}$ Yamauchi $\mathrm{H}$, Kito $\mathrm{H}$, Uda K: Studies on Intraocular Penetration and Metabolism of Fluromethalone in Rabbits: A Comparison Between Dexamethasone and Prednisolone Acetate. Jap J Ophthalmol 1975, 19: 339-47.

${ }^{13}$ Kupferman A and Leibowitz HM: Biological Equivalence of Ophthalmic Prednisolone Acetate Suspensions. Am J Ophthalmol 1976, 82: 109-13. 
${ }^{14}$ Leibowitz HM and Kupferman A: Kinetics of Topically Administered Prednisolone Acetate. Arch Ophthalmol 1976, 94: 1387-9.

${ }^{15}$ Flint GR and Morton DJ: Effect of Derivatization on the Bioavailability of Ophthalmic Steroids: Development of an In Vitro Method of Evaluation. Arch Ophthalmol 1984, 102: 1808-9.

${ }^{16}$ Watson D, Noble MJ, Dutton GN, Midgley JM, Healey TM: Penetration of Topically Applied Dexamethasone Alcohol into Human Aqueous Humour. Arch Ophthalmol 1988, 106: 686-7.
${ }^{17}$ Midgley JM, Watson DG, Healey T, Noble M: The Quantification of Synthetic Corticosteroids Using Isotope Dilution Gas Chromatography Negative Chemical Ionisation Mass Spectrometry. Biomed and Environ Mass Spectrom 1988, 15, 479-83.

${ }^{18}$ Krupin T, Waltman SR, Becker B: Ocular Penetration in Rabbits of Topically Applied Dexamethasone. Arch Ophthalmol 1974, 92: 312-14.

${ }^{19}$ Southern AL, Altman K, et al: Steroid Metabolism in Ocular Tissues of the Rabbit. Invest Ophthalmol 1976, 15: 222-7. 